\title{
Proposta de um Sistema de Apoio à Doação Sanguínea Baseado em Gamificação
}

\author{
Proposal of a System to Support Blood Donation Based on Gamification
}

\author{
Raniel Gomes da Silva ${ }^{1}$ (i) http://orcid.org/0000-0003-4874-3447 \\ Danilo Ricardo Barbosa de Araújo ${ }^{1}$
}

${ }^{1}$ Escola Politécnica de Pernambuco, Universidade de Pernambuco, Recife, Brasil

E-mail do autor principal: Raniel Gomes da Silva raniel90@gmail.com

\section{Resumo}

Os diversos meios de comunicação abordam constantemente reportagens sobre o baixo nível no estoque de sangue no estado de Pernambuco; também são divulgadas diversas campanhas de conscientização para que a população realize uma doação de sangue e auxilie pacientes em déficit sanguíneo. Geralmente essas campanhas se intensificam quando algum período festivo ou um feriado prolongado estão próximos, tudo isso porque, de acordo com diversas estatísticas, as incidências de acidentes aumentam e consequentemente a demanda por sangue para serem usados em cirurgias, transfusões, etc. Este artigo tem como objetivo apresentar o projeto de um software, denominado 1 updonate, desenvolvido com o objetivo é ser um novo canal de apoio aos doadores. A arquitetura do sistema é dividida em dois módulos principais, um voltado para os doadores, e o outro para a manutenção dos bancos de sangue. O principal diferencial do sistema proposto é o uso da gamificação, que possui potencial para promover um maior engajamento dos doadores no processo de doação.

Palavras-Chave: Doação de Sangue; Gamificação; Hemocentro; Aplicação Móvel; Aplicação Web.

\begin{abstract}
The several communication channels present constantly reports and news about the low level of blood stock in the state of Pernambuco; also there are several campaigns in order to raise awareness of the population towards blood donation and assist patients in blood deficits. Often these campaigns are intensified when some festive period or an extended holiday are close, all this because, according to various statistics, accident incidences increase and consequently the demand for blood to be used in surgeries, transfusions, etc. This paper aims at presenting the project of a software, called 1updonate, developed with the objective of being a new channel to support donors. The system architecture is divided into two main modules, one facing the donor and the other for the maintenance of blood banks. The main distinguishing feature of the proposed system is the use of gamification and its ability in promoting the engagement from donors in the whole process.
\end{abstract}

Key-words: Blood Donation; Gamification; Blood Center; Mobile Application; Web Application. 


\section{Introdução}

Em meados da década de 80 , era bastante comum que as doações de sangue fossem remuneradas para garantir o suprimento necessário dos hemocentros com mais eficiência. Todavia, após a propagação demasiada de doenças sexualmente transmissíveis, como a AIDS, a Organização Mundial de Saúde (OMS) vetou qualquer tipo de comercialização do sangue, com o objetivo de evitar a proliferação ainda maior destas doenças (Gonçalez et al., 2003).

Após a proibição de remuneração em doações de sangue, a iniciativa pública ficou responsável por garantir o recrutamento de novos doadores. $\mathrm{Na}$ década de 90 , foi instaurado o mecanismo de doação de reposição, cujo objetivo é armazenar nos hemocentros o estoque restante em que o doador disponibilizou para auxiliar algum familiar ou amigo (Barboza, Stephanie Ingrid Souza, 2014). Apesar dessa técnica de reposição ser bastante aplicável, ela não garante que os estoques sanguíneos se mantenham em seu estado ideal; existem diversos fatores que continuam dificultando o canal entre os hemocentros e os doadores. De acordo com o Ministério da Saúde, 1,8\% da população brasileira doa sangue e, desses doadores, mais de $50 \%$ são espontâneos. Entre 2013 e 2014, houve um aumento de $5 \%$ na coleta de bolsas de sangue no país, passando de 3,5 milhões para 3,7 milhões (OMS, 2016). Ainda assim, existe a preocupação em sensibilizar e fidelizar novos doadores.

A falta de informação do processo, o histórico referente aos casos de aquisição da AIDS através da transfusão sanguínea e o baixo grau de instrução educacional, leva o indivíduo a ter receio de realizar o processo. A renda per capita também é um fator relevante, pois segundo a OMS, das 108 milhões de doações recolhidas anualmente, metade são em países de alta renda, o que corresponde a apenas $20 \%$ da população mundial (OMS, 2016). Outro fator recente, que tem dificultado o engajamento de doadores, é o aumento no índice de sangue contaminado devido à infecção de arboviroses, como a zika, chikungunha e dengue.

Atualmente, existem diversos sistemas disponíveis, voltados para o doador, com relação ao atendimento das demandas citadas, como por exemplo as ferramentas: o Sangue Social (Moraes; Moreira, 2015) e o Blood Donor (Software blood, 2016). Porém as ferramentas disponíveis possuem deficiências com relação à adequação do processo ao contexto Pernambucano, onde haja mecanismos para engajamento do usuário e do fluxo de doação de sangue no próprio software.
Este artigo tem como objetivo apresentar o projeto de um sistema desenvolvido que visa aproxime o doador dos hemocentros. O doador pode baixar o aplicativo na loja de apps, se cadastrandoatravés do login do Facebook. A partir desse momento poderá ir ao hemocentro mais próximo realizar a doação. Após este fluxo, o doador fará o check-out do banco de sangue, o sistema validará a sua ação e caso seja válido, os dados deste doador serão utilizados para engajá-lo através de integração gamificada com outros doadores e notificações periódicas sobre campanhas sanguíneas. Os dados gerados serão visíveis através de um portal para que os funcionários dos hemocentros acompanhem como está o fluxo de doação. Neste painel também será possível cadastrar os hemocentros, estoques sanguíneos e campanhas.

Este artigo está organizado da seguinte forma. A seção 2 apresenta a visão geral sobre a gamificação e a Seção 3 detalha a metodologia usada no trabalho. Já a Seção 4 descreve o sistema desenvolvido e a Seção 5 apresenta trabalhos relacionados. Por fim, a Seção 6 apresenta a conclusão e trabalhos futuros.

\section{Visão Geral sobre Gamificação}

A gamificação foca na resolução de problemas do dia-a-dia e no maior engajamento do usuário através da dinâmica de jogos (Vianna et al., 2015). A aplicação em ambientes corporativos é um forte exemplo que pode utilizar este conceito, pois pode contribuir no aumento de desempenho dos colaboradores, a partir da mudança de técnicas e metodologias que desmotivam a sua utilização, por serem entediantes e não atrativas. Essa agregação torna-se vantajosa para as empresas, pois a maioria das pessoas que jogam, possuem esta prática desde a infância (Vianna et al., 2015). De forma simplificada pode se dizer que os jogos virtuais têm como principal foco trazer o entretenimento e a diversão, já a gamificação utiliza os benefícios do mundo digital como ferramenta de engajamento para as aplicações que a embarcam (Stadler; Bilgram, 2016). Existem diversos aplicativos com diferentes mecanismos que utilizam essa visão. A Nike + FuelBand utiliza um software mobile e uma pulseira com tecnologia embarcada para realizar o monitoramento das atividades dos usuários (Nike, 2016). O FourSquare possibilita a interação humano computacional através dos check-ins (FourSquare, 2016). O RunKeeper acompanha as corridas, caminhadas, dentre outros esportes, para informar ao esportista como está o seu progresso e desempenho (RunKeeper, 2016). Para que haja uma aplicação eficiente da gamificação é necessário que as tarefas sejam orientadas por metas 
claras, possíveis de serem executadas e que fomentem 0 interesse do usuário. 0 jogo deve informar o resultado ao participante através de um constante sistema de feedback (McGonigal, 2012). O sistema de feedback agrupa uma série de gratificações ao jogador: através dos alcances obtidos (Achievements), status (distribuição de badges ou medalhas, e ranking), acesso (disponibilização de funcionalidades secretas) e presentes (giftings) (Vianna et al., 2015). O usuário deve participar voluntariamente das atividades e não deve ser forçado, ou seja, deve haver uma comunicação clara e consentimento mútuo entre o jogo e o jogador. Só haverá um ambiente gamificado se o usuário concordar com toda essa estrutura definida (Vianna et al., 2015). Cada jogador tem um objetivo específico, e conceitualmente é possível classificar os possíveis objetivos em quatro categorias: predador, realizador, explorador e socializador. Os predadores têm como perfil derrotar adversários; já os conquistadores visam atingir metas; os exploradores focam na investigação ou estudo dos recursos do jogo; por fim os socializadores desbravam o jogo junto com outros participantes (Vianna et al., 2015). Em (Stadler et al., 2016) os autores evidenciam as melhores práticas de gamificação dentro de dois contextos: na pesquisa de mercado e no turismo. No estudo de caso da pesquisa de mercado são abordadas as dificuldades quanto há necessidade de se realizar pesquisas muito detalhadas; questionários grandes e detalhados acabam deixando as pessoas cansadas de responder atentamente a todas as perguntas. Assim, foi criado um jogo para identificar as necessidades do consumidor; se determinado produto corresponde às necessidades do cliente, o mesmo aplica um pareamento para o recurso. Já no emprego da gamificação no turismo, o usuário é engajado para compartilhar as experiências de viagens e fotos dos locais.

\section{Metodologia}

Para o desenvolvimento deste artigo foi utilizada a metodologia descrita a seguir.

Inicialmente foi feita uma revisão da literatura, contemplando artigos voltados para o contexto de mobilidade, redes sociais e deficiências no processo de gestão e captação de doadores. Essa revisão também se baseou na pesquisa de livros relevantes e conceituados na área de gamificação: (McGonigal, Jane; 2012) e (Vianna et al., 2015).
O levantamento dos trabalhos relacionados tomou como base pesquisas nas ferramentas on-line Google Scholar e o Portal de Períodico da CAPES. As strings de busca incluíram: "blood donation application", "blood donation application" AND "blood center", "gamification" AND "blood donation" e "mobile application" AND "gamification" AND "blood". As pesquisas foram realizadas no período de Junho a Julho de 2016, com ênfase nos trabalhos recentemente publicados.

Após esta etapa de entendimento do contexto de atuação da proposta, foi delimitado o escopo do trabalho, possibilitando o estudo de viabilidade para que a pesquisa pudesse se tornar em software funcional.

Para o desenvolvido de um protótipo, foi necessário aplicar conhecimentos de diversas disciplinas da área de engenharia de software. 0 processo adotado consistiu dos seguintes passos: elicitação, especificação e validação de requisitos (Pohl; Rupp, 2013), implementação de software, testes orientados para ambientes web. Foram usadas linguagens e tecnologias conhecidas para desenvolvimento de projetos OO, tais como: UML (Linguagem de Modelagem Unificada) através dos casos de uso (Pressman, 2013), linguagem de programação Java e modelagem de banco de dados (Elmasri, Navathe; 2010).

Como no âmbito do levantamento de requisitos é primordial a participação de stakeholders, foi realizada uma reunião com gerente de tecnologia de informação do HEMOPE (Hemocentro de Pernambuco). O objetivo dessa reunião foi identificar - que poderia ser desenvolvido no protótipo da ferramenta. Os pontos chave levantados foram: a pontuação dos doadores através de identificadores no cartão de doação, ou seja, gamificar um serviço para prover uma motivação ao doador, e uma estratégia de maior aproximação com esses usuários. A partir disso, o projeto web e mobile foi delineado com o propósito de estruturar uma arquitetura, cujas funcionalidades provessem os requisitos levantados pelo colaborador do HEMOPE.

A arquitetura do sistema foi projetada logo após a definição do que seria implementado. O tipo de arquitetura foi o cliente-servidor, cujo objetivo é centralizar 0 processamento das requisições solicitadas pelos dispositivos móveis.

Por fim, estruturou-se as funcionalidades do software. $O$ projeto agrupou os requisitos funcionais de cadastro de hemocentros, campanhas de doação,

http://dx.doi.org/10.25286/repa.v2i2.553 
estoque sanguíneo (web), processo de doação, listagem de hemocentros, campanhas de doação e estoque sanguíneo, visualização dos pontos obtidos e ranking (mobile). Todos estes requisitos foram implementados, com exceção do relatório de campanhas por usuário e por data no ambiente web.

\section{Plataforma Proposta}

O sistema 1upDonate possui dois módulos principais, um voltado para os administradores dos centros de sangue e o outro voltado para os usuários finais, ou seja, os doadores. Através do portal, podem ser cadastrados os hemocentros, filiais dos hemocentros, estoques sanguíneos e campanhas para doação. Todos estes dados serão disponibilizados para a aplicação móvel através de serviços REST. No lado do cliente, estes dados são recebidos e armazenados localmente na inicialização da aplicação A Figura 01descreve os módulos da estrutura proposta.

O sistema 1upDonate utiliza os princípios de achievements, status e socialização para promover engajamento e aumentar o interesse dos usuários por doações. Os achievements são modelados através de corações, representando diferentes níveis de engajamento no sistema de doações (por exemplo, com o uso de corações dourados e de diamante).

Os status dos achievements são definidos como Doador Solidário para quem fez pelo menos uma doação na vida; Doador Veterano para quem tem mais de 3 corações; Doador Campeão para quem tem mais de 6 corações. O doador que conseguir outros doadores terá o status de Capitão de Doações. Nas Figuras 09 e 10, pode se observar como os elementos de achievements e status, são mostrados no aplicativo.

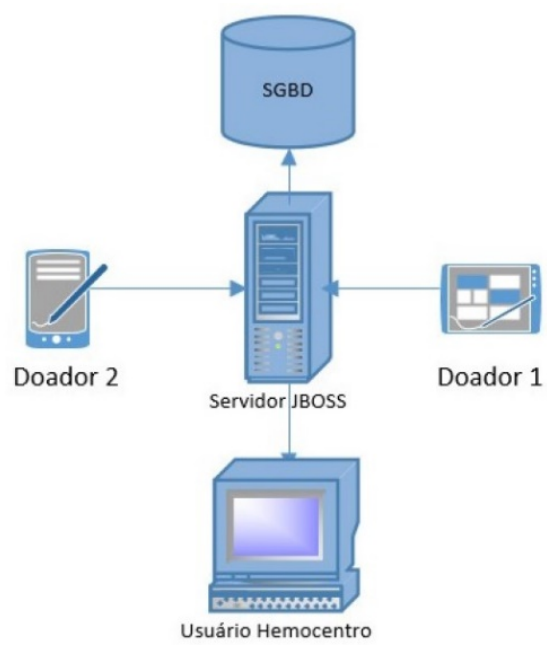

Figura 1: Arquitetura da Aplicação.

Com relação ao acesso todos os recursos serão disponíveis para todos os usuários. A utilização de giftings não foi implementada, uma vez que a ideia principal do processo de doação é de que seja totalmente voluntária e não remunerada. O 1 upDonate é disponibilizado para um único tipo de jogador, com as classificações mescladas de explorador e socializador.

\subsection{Requisitos Funcionais}

Os requisitos funcionais do módulo web da aplicação 1upDonate são: realizar login, manter hemocentro, manter filiais dos hemocentros, manter estoques sanguíneos, manter campanhas, manter usuário, manter grupos de usuário, manter perfil de usuário, enviar hemocentros, enviar filiais dos hemocentros, enviar estoques sanguíneos e enviar campanhas.

Já os requisitos funcionais do módulo mobile são: realizar login pela rede social Facebook, receber hemocentros, receber filiais dos hemocentros, receber estoques sanguíneos, receber campanhas, visualizar dashboard do doador, visualizar ranking dos doadores, detalhar campanha, detalhar hemocentro, compartilhar campanha sanguínea, visualizar estoque sanguíneo e visualizar localização do hemocentro.

\subsection{Requisitos Não-Funcionais}

Os requisitos não funcionais do módulo web são: segurança dos dados através da autenticação obrigatória do usuário para acesso aos dados, segurança do códigofonte, pois toda a regra de negócios foi injetada no back-end e segurança do trafégo de dados utilizando o protocolo https. 
Escalabilidade do sistema ao aplicar o conceito de servidores virtualizados, ou seja, à medida que aumenta a demanda de hardware para o processamento das requisições, é possível parametrizar o uso de memória e processamento. Usabilidade ao aplicar um conceito genérico nos layouts, garantindo um fácil aprendizado da navegabilidade do sistema. Os requisitos não funcionais do módulo mobile são: baixo consumo de dados do dispositivo através do mínimo possível de requisições ao servidor, segurança de dados para não permitir que o usuário não acesse além do disponível para o seu perfil, usabilidade a partir de padrões de componentes CSS e HTML para plataformas híbridas e bom desempenho a partir de um desenvolvimento a partir da aplicação correta dos conceitos da linguagem MVC AngularJS.

\subsection{Casos de Uso}

A Tabela 01 descreve as ações possíveis que o usuário do portal poderá realizar. Esses recursos serão exclusivamente para funcionários dos hemocentros. Já a Tabela 02 descreve o caso de uso para o ator sistema, cujas ações disponíveis, são os serviços web que serão requisitados pela aplicação mobile. Por fim, a Tabela 03 descreve a visão funcional do ator doador. Todas estas ações estão disponíveis no aplicativo mobile.

Tabela 1: Casos de uso usuário portal

\begin{tabular}{c|}
\hline Acessar sistema \\
\hline Manter hemocentro \\
\hline Manter filiais dos hemocentros \\
\hline Manter estoque sanguíneo \\
\hline Manter campanhas de doação \\
\hline Manter usuário
\end{tabular}

Tabela 2: Casos de uso serviços REST.

\begin{tabular}{c|}
\hline Receber doações realizadas \\
\hline Sincronizar campanhas \\
\hline Sincronizar filiais dos hemocentros \\
\hline
\end{tabular}

51

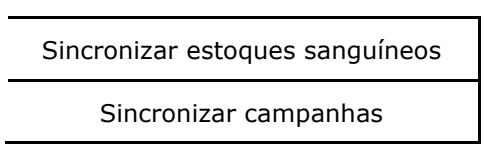

Tabela 3: Casos de uso do Doador.

\begin{tabular}{c|}
\hline Realizar login \\
\hline Receber hemocentros \\
\hline Receber campanhas \\
\hline Visualizar dashboard \\
\hline Visualizar ranking dos doadores \\
\hline Detalhar campanha \\
\hline Detalhar Hemocentro \\
\hline Visualizar estoque sanguíneo \\
\hline Visualizar localização do hemocentro
\end{tabular}

\subsection{Protótipo do Módulo para Doadores}

No ecossistema da aplicação móvel, o doador deve começar pelo login. De acordo com a Figura 2, 0 acesso é feito apenas pela rede social Facebook:

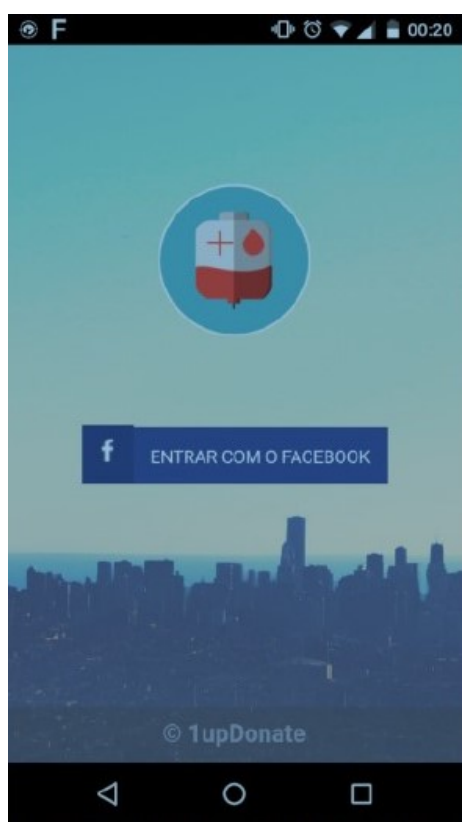

http: / / dx.doi.org/10.25286/repa.v2i2.553 
Figura 2: Login através do Facebook.

Por padrão, o plugin utilizado para login pelo Facebook, redireciona o sistema para outra tela. Conforme a Figura 3, o doador deverá digitar o seu usuário e senha desta rede social.

Após o login, o doador será redirecionado para o dashboard, conforme a Figura 4. Nesta tela, são exibidas de forma resumida, as últimas campanhas cadastradas. Ao clicar em alguma campanha, o sistema exibirá os detalhes da pessoa que precisa do sangue. Caso o doador atenda a necessidade de algum solicitante, a pontuação obtida será maior que a doação padrão (por hemocentro).

$\mathrm{Na}$ Figura 5, os hemocentros são exibidos conforme cadastro no portal. Ao clicar em um banco de sangue, a Figura 6 mostra os detalhes do hemocentro selecionado, como endereço e estoque sanguíneo. Na mesma tela é possível iniciar o processo de doação, como também visualizar o endereço no mapa.

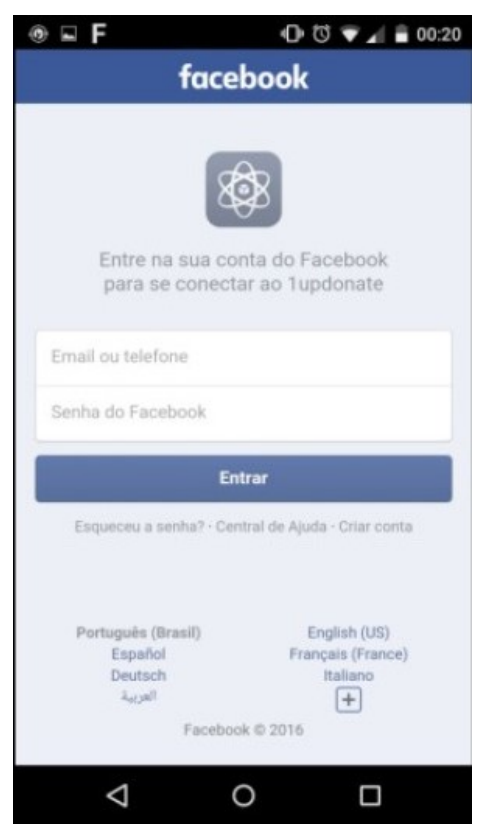

Figura 3: Formulário de login.

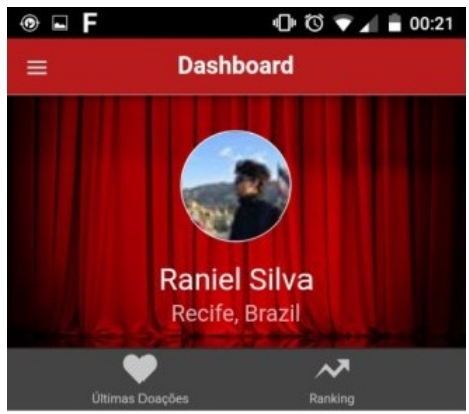

Últimas campanhas

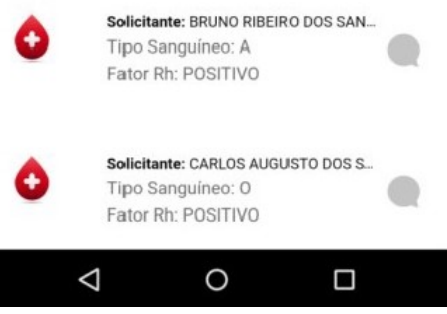

Figura 4: Dashboard do doador.

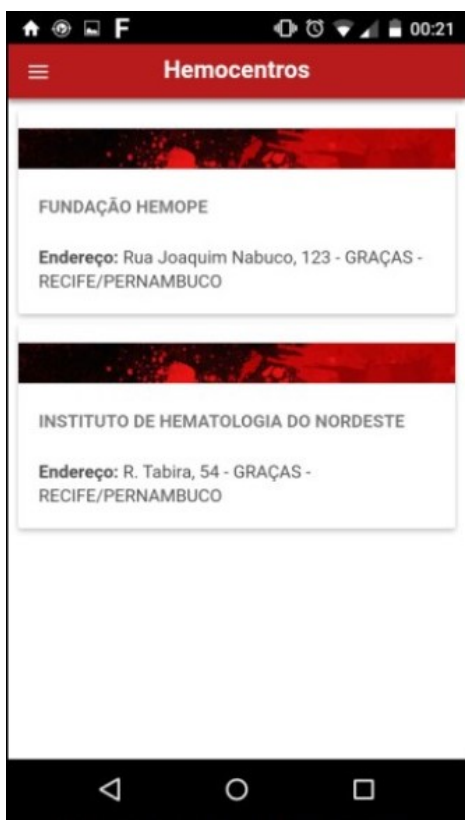

Figura 5: Listagem de hemocentros. 


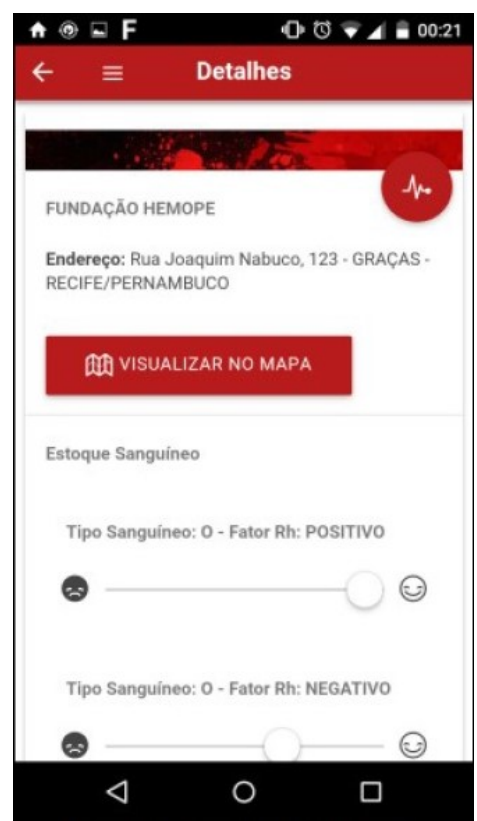

Figura 6: Detalhes do hemocentro.

Ao iniciar o processo de doação, basta clicar no botão circular que está no lado superior direito, conforme Figura 6. A cada processo de doação, são passadas algumas instruções para que o doador verifique se suas condições de saúde estão aptas para o processo, de acordo como é informado na Figura 7.

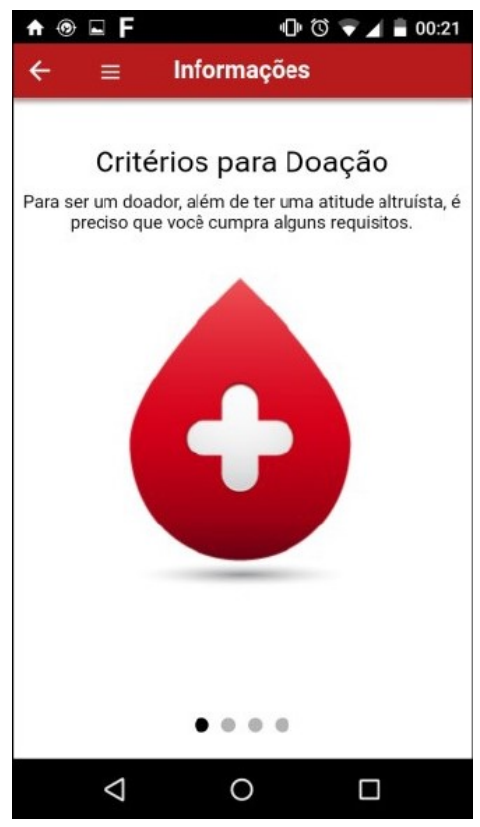

Figura 7: Critérios de doação (página 1).
Um mapa com local do cliente é exibido na Figura 8. Ao clicar no marcador vermelho, o sistema exibe um maior detalhamento do endereço.

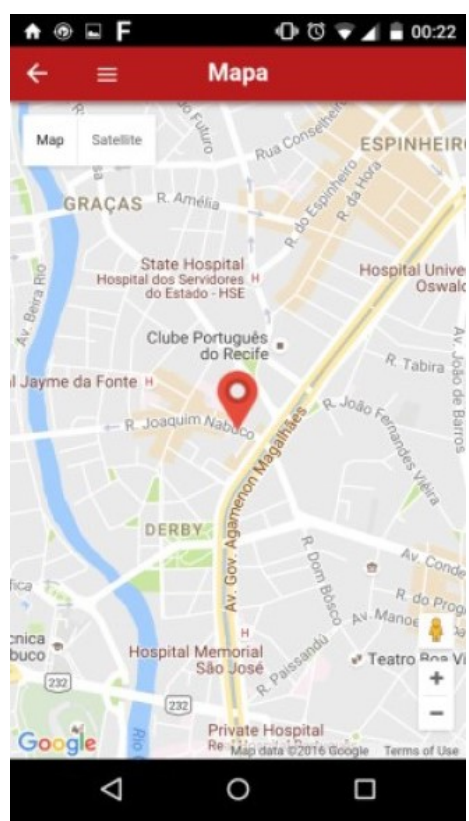

Figura 8: Visualizar mapa.

Caso ele realmente queira validar essa atividade, a Figura 9 mostra um exemplo para o doador prosseguir com fluxo. O objetivo dessa ação é de validar a geolocalização do usuário e se a vigência é válida de acordo com a última data de doação. 


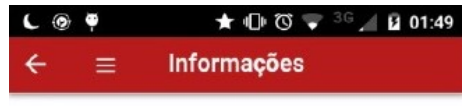

Qualquer critério para a doação pode ser alterado pelo triagista, a quem cabe, de acordo com a análise do candidato à doação, a autorização final.

Para realizar sua doạ̧ăo, clique no botẫo abaixo:

\section{REALIZAR DOAÇĀo}

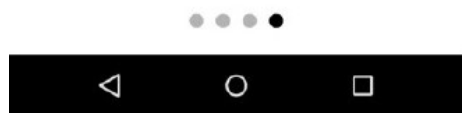

Figura 9: Realizar doação.

Toda transfusão sanguínea realizada é computada como pontuação para o doador. Esses dados serão exibidos no perfil do usuário. Será também possível visualizar quais são os alcanços (achievements) em percentual, nível e quantidade de corações (badges). Além disso, o sistema periodicamente lançará algumas atividades extras que podem valer corações extras caso o usuário consiga atender o especificado. Essas tarefas são avisadas via notificação push (push notification, 2016) e exibidas no perfil conforme Figura 10.

O ranking dos usuários mais ativos no 1upDonate, pode ser acessado via menu lateral ou pelo dashboard (Figura 4). Conforme a Figura 11, é possível identificar no ranking qual o tipo do doador, suas conquistas e se o mesmo também é um capitão de doações. Essa funcionalidade é bastante interessante pois, de certa forma, motiva as pessoas a participarem neste processo solidário. Nessa tela, o usuário também pode compartilhar os seus dados no Facebook.

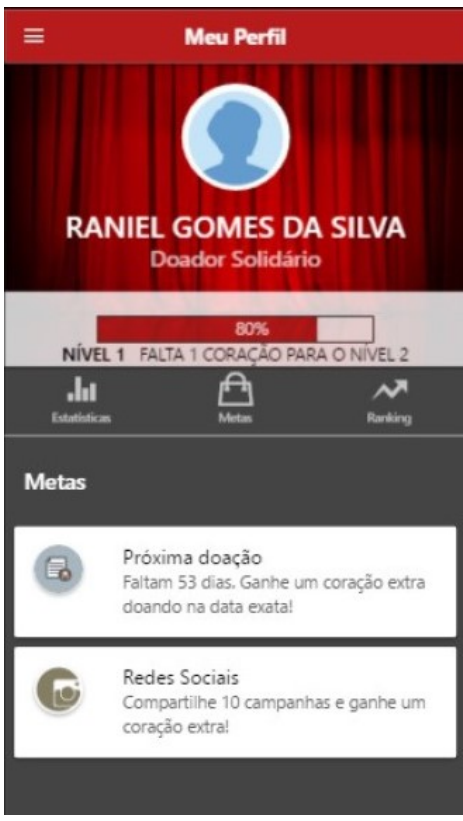

Figura 10: Dashboard gamificado do usuário.

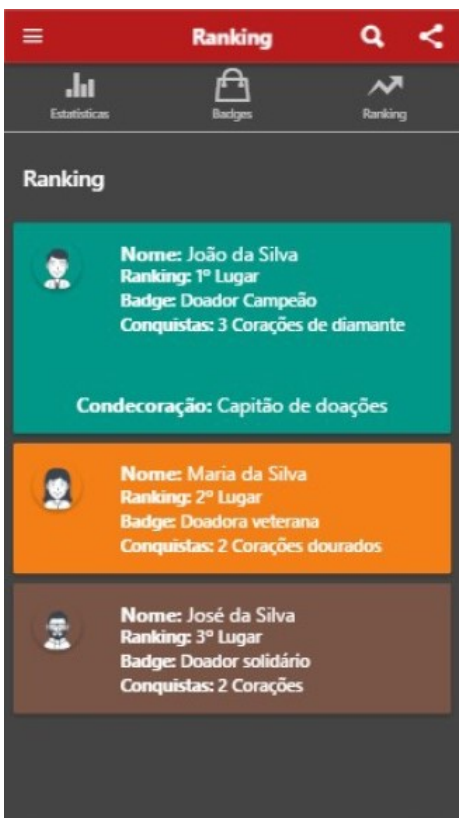

Figura 11: Dashboard gamificado do usuário.

\subsection{Tecnologias Utilizadas}

Esta seção descreve as tecnologias mobile e web utilizadas para a implementação do sistema 1 upDonate.

A aplicação móvel foi desenvolvida através da tecnologia Córdova. Para o layout, foi utilizado o 
framework Ionic. Os dados do usuário são armazenados localmente através do SQLite. Ao gerar um executável da versão implementada, é possível testar nos sistemas operacionais Android, iOS e Windows Phone, no entanto, a build de cada versão possui suas nuances. No caso do iOS, por exemplo, é necessário utilizar o XCode em um dispositivo Apple como por exemplo um MacBook e após isso compilar, pois o Windows não permite tal atividade (Apple XCode, 2016).

O sistema web foi desenvolvido em Java EE com JPA, JSF, Primefaces, Jboss EAP e JAX-WS. A robustez e escalabilidade com o Java são pontos cruciais que irão garantir maior estabilidade e segurança da ferramenta web em produção (Robustez Jboss, 2016).

\subsubsection{Apache Cordova}

Para tornar a aplicação operável em diversos dispositivos móveis e para garantir isso, foi necessário implementar as mesmas funcionalidades em diferentes sistemas operacionais, tornando a curva de aprendizado maior, além dos gargalos em relação à manutenção e melhorias, pois seria necessário pensar nos mecanismos programáticos para cada plataforma. Com o cordova é possível construir aplicações híbridas utilizando HTML, CSS e JavaScript, sendo possível disponibilizar versões para Android, iOS, Windows Phone, BlackBerry, entre outras plataformas móveis e web, como Ubuntu e Windows.

\subsubsection{NodeJS}

Com o aumento da utilização de plataformas híbridas com HTML, CSS e JavaScript, cresceu bastante o número de bibliotecas para auxiliar os desenvolvedores a criarem interfaces mais ricas e arquiteturas mais consistentes. O gerenciamento dessas dependências pode se tornar uma tarefa trabalhosa e para isso, foi utilizado o NodeJS, cuja finalidade é garantir o gerenciamento desses pacotes através do ecossistema NPM (Node Package Manager), na qual permite o download e atualizações dessas bibliotecas de maneira prática e simples.

\subsubsection{AngularJS}

Aplicações híbridas geralmente precisam que as regras de negócio sejam implementadas com Java
Script e de alguma forma, as solicitações e retornos precisam ser claros para os usuários. Para tornar esse processo mais enxuto, o framework de componentes web AngularJS foi utilizado. Ele é responsável por permitir uma maior dinâmica e controle para a camada de apresentação, além de possuir uma biblioteca em seu núcleo que contém componentes HTML customizados para o padrão do Angular]S.

\subsubsection{Ionic}

Devido à alta exigência dos usuários em relação a aplicações móveis, é necessário que a aplicação além de ser multiplataforma e robusta em termos de regras de negócio, seja também atraente e elegante. 0 AngularJS possui uma integração bastante compatível com o framework Ionic. Essa tecnologia é capaz de criar interfaces ricas, cujo layout atende com excelência, os fatores de responsividade e consistência dos seus recursos, além de possuir uma documentação bastante clara, de fácil entendimento e de contínuas atualizações.

\subsubsection{JavaEE}

Java é uma linguagem de programação orientada objetos e possui interoperabilidade nas versões padrão, micro e empresarial. A versão utilizada em ambientes web é a empresarial, pois garante uma maior gama de recursos para serem implementados sem a necessidade de incluir dependências de terceiros. Algumas das tecnologias que existentes no java EE e que serão utilizadas no projeto são oEJB, JPA e JSF.

Enterprise Java Beans é uma estrutura utilizada dentro da arquitetura java EE para o desenvolvimento de componentes de negócio distribuídos, seguros, transacionais e reutilizáveis. A implementação das regras de negócio é realizada nessa camada e permite que diversos clientes acessem o mesmo EJB, pois 0 conceito de escalabilidade também é aplicado.

Para o gerenciamento dos dados, foi utilizado o framework de mapeamento objeto relacional JPA (Java Persistence API), pois garante uma fácil implementação e manutenção. Com o JPA, o desenvolvedor preocupase apenas em realizar a modelagem através das classes básicas dentro do projeto Java e ao iniciar o servidor de aplicação, é

http://dx.doi.org/10.25286/repa.v2i2.553 
possível criar ou alterar a estrutura de definição das tabelas e campos da base de dados.

Para tornar o desenvolvimento de telas mais eficaz e produtivo, a partir da versão 6 do java $E E$, foi disponibilizado um novo framework de componentes web. O JSF tem sido bem aceito por empresas e estudantes, devido a sua robustez e fácil integração com API's para telas, como IceFaces, RichFaces e Primefaces. Ao contrário do JSP, o JSF possui uma sintaxe própria, pois suas tags são bem específicas. Para utilizá-las, basta apenas importar a biblioteca de tags e implementá-las em arquivos XHTML.

Para que as interfaces (telas) tenham um design atraente e elegante, 0 framework Primefaces foi integrado ao JSF. Ele possui total relação com a biblioteca do JQueryUI e permite abertura para customizações de CSS e JavaScript do seu núcleo. Esta tecnologia possui uma implementação para a maioria dos componentes do JSF, sendo necessário apenas importar a biblioteca de tags e definir os componentes do Primefaces.

\subsubsection{Outras Tecnologias Usadas}

O apache Maven é uma ferramenta para o gerenciamento de dependências, construção e plugins para projetos Java e outras linguagens. A sua configuração é feita através de um arquivo em XML e nele contém todos blocos citados acima. Ao instalar o Maven em um projeto, todas as bibliotecas declaradas no arquivo XML (POM - modelo de objetos do projeto) são baixadas para um diretório local do usuário.

O Jboss é um servidor de aplicação de código-fonte aberto, responsável pela execução de softwares java web. O objetivo dele é garantir a execução de uma aplicação com alta disponibilidade, escalabilidade e bom desempenho. A versão EAP (plataforma de aplicações empresariais) possui certificação que compatibiliza com as especificações definidas na arquitetura java EE.

Para prover a comunicação através de webservices entre as aplicações cliente e servidor, o framework de REST (transferência de estado representacional) resteasy, que por sua vez, possui por padrão no servidor de aplicação Jboss, cuja implementação é baseada na especificação JAX-RS da arquitetura JavaEE.

O Postgres é um sistema de gerenciamento de banco de dados com uma estrutura objeto-relacional. Este SGBD é de código-fonte livre e sofre constantes evoluções e melhorias para que as aplicações tenham um melhor controle dos dados e de suas transações.

\section{Trabalhos Relacionados}

Esta seção contém um resumo do levantamento dos sistemas existentes no nicho de doações de sangue e engajamento do usuário; também são observados os possíveis gargalos destas ferramentas. São analisados os seguintes trabalhos: Aplicação para gestão de hemocentros (Moraes; Moreira, 2015); Um modelo ubíquo para doação de sangue (Colling et al., 2014); Pesquisa de uma comunidade de doadores a partir de dispositivos móveis (A Bhowmik et al., 2015); Doação de sangue em sistemas em nuvem (Fotopoulos et al., 2016); Aplicações móveis gratuitas para doação de sangue (Ouhbiet al., 2015); Design e desenvolvimento de um rastreador móvel para doação de sangue (S. Abu Naser et al., 2016).

\subsection{Aplicação para Gestão de Hemocentros}

Em 2015, Éder Moraes e Rossana Moreira desenvolveram a aplicação Sangue Social, cuja finalidade é propagar campanhas que poderão ser divulgadas através do Facebook com o objetivo de captar, fidelizar e organizar os cadastros dos doadores. As tecnologias utilizadas foram o PHP, MySQL, Api Facebook Developers, HTML, CSS e JQuery. A necessidade de criar a aplicação Sangue Social foi gerada devido ao baixo índice de doadores regulares no Brasil; além disso, os canais de comunicação não são eficientes para a propagação das campanhas. Ela foi desenvolvida só para a web, tornando-se limitada em relação às plataformas existentes, uma vez que a quantidade de usuários em dispositivos móveis tem aumentado muito nos últimos anos.

\subsection{Um Modelo Ubíquo para Doação de Sangue}

Em 2014, Ilseu Colling, Cristiano Costa e Rodrigo Righi, escreveram um artigo relacionado a um modelo ubíquo para doação de sangue, e apresentaram a estrutura de um sistema que através das redes sociais, permite divulgar as doações de acordo com a geolocalização do doador. Este artigo é uma extensão do Sangue Social, mas foi desenvolvida por outros autores.

A lei no 10.205/2001, conhecida como Lei do Sangue (Lei Betinho), diz que o processo de doação 
de sangue deve ser espontâneo, solidário e não remunerado (Lei do Sangue, 2001).

Em relação à pesquisa o autor deixa claro que não foi encontrado nenhum artigo voltado para a computação ubíqua com redes sociais para o processo de doação de sangue. Em relação às funcionalidades do software, o usuário não recebe notificações no dispositivo móvel e a aplicação foi desenvolvida apenas para o sistema operacional Android, sem contar que a versão utilizada (2.1) está bem abaixo da versão atual (6.0 - Marshmallow), fazendo com que o layout e demais melhorias desenvolvidas pela comunidade Google, estejam bastante ultrapassadas.

\subsection{Pesquisa em uma Comunidade de Doadores a Partir de Dispositivos Móveis}

Este trabalho é relacionado à pesquisa de uma comunidade de doadores a partir de dispositivos móveis (A Bhowmik et al., 2015). Ele foca na diminuição da lacuna, entre as pessoas que querem doar e as que precisam de sangue em caso de emergências, através do uso de processos tecnológicos.

Porém, os autores não abordam a proposta de implementação em si, apenas detalham que será criada para diversas plataformas móveis. Além disso, são feitas algumas pesquisas com alguns usuários da cidade Bangladesh, com o objetivo de mapear as questões sociais e o engajamento tecnológico dos usuários para entender se a criação do software é viável ou não. Neste país, existe uma grande carência no recrutamento de novos doadores e na atualização dos dados cadastrais destes, sem contar que existem poucos sites quem fornecem um auxílio para esse controle. Em relação às possíveis funcionalidades, há um recurso interessante: um botão de pânico para o que o usuário já alerte as entidades médicas, para que seja alocada alguma bolsa de sangue para o seu tipo sanguíneo.

\subsection{Doação de Sangue em Sistemas em Nuvem}

Os sistemas em nuvem para doação de sangue não são muito diferentes dos anteriores citados porém, trazem um dado interessante. Em 2050, é esperado que o número de pessoas com 65 anos ou mais de idade quase triplique em cerca de 1,5 bilhões, representando 16 por cento da população mundial (Fotopoulos et al., 2016). Isso acarreta no aumento da restrição da elegibilidade do doador e no aumento de procedimentos oncológicos e cirúrgicos que demandam muito sangue nestes processos.

Um ponto positivo da estratégia dos sistemas em nuvem citada por Ilias Fotopoulos e demais autores é o mecanismo para identificar o potencial e a elegibilidade dos doadores. Ao reservar ou agendar uma doação, o ambiente web recebe essa requisição e dispara um web service para analisar o quadro clínico do doador, fazendo com que o processo de triagem seja feito de forma mais rápida e menos burocrática.

\subsection{Aplicações Móveis Gratuitas para Doação de Sangue}

O trabalho relacionado ao estudo de diferentes aplicações moveis voltadas para doação de sangue (Ouhbi et al., 2015), tem como foco mensurar as características positivas e negativas dos softwares dentro do contexto da saúde com ênfase na doação de sangue. Esse artigo auxiliou bastante no levantamento de outros produtos e na identificação das melhores técnicas e recursos a serem utilizadas para garantir o máximo de aceitação possível pelos usuários.

\subsection{Design e Desenvolvimento de um Rastreador Móvel para Doação de Sangue}

O último trabalho aborda o design e desenvolvimento de um rastreador móvel para doação de sangue (S. Abu Naser et al., 2016). Essa ferrramenta é capaz de conectar-se a um hemocentro para facilitar a coleta de sangue dos usuários em caso de emergência e facilitar a comunicação dos doadores entre si e entre os hemocentros.

O sistema a ser criado é limitado às plataformas Android. $O$ artigo foca no contexto técnico e não no negócio.

No caso de uso do software, os usuários são cadastrados através do portal; isto torna a manutenção custosa, pois esse processo poderia ser

http://dx.doi.org/10.25286/repa.v2i2.553 
feito automaticamente, no momento em que o usuário realiza o processo de autenticação no sistema.

\subsection{Considerações sobre os Trabalhos}

Nos projetos de referência abordados, é possível identificar as seguintes deficiências: a maioria deles não vislumbra um mecanismo para a captação de novos doadores ou no engajamento dos usuários existentes. Apenas o Sangue Social, agrega a funcionalidade de integração com as mídias sociais. A interoperabilidade das aplicações para os sistemas operacionais iOS e Windows Phone é pouco aplicada, sendo a maioria voltada para Android e web. Apenas o artigo de Dorothe Stadler e Volker Bilgram mensura o tópico de gamificação para potencializar a usabilidade e utilização da ferramenta, pois seu foco é totalmente diferente da doação de sangue.

\section{Conclusões}

A partir da revisão da literatura, observou-se que existem diversos gargalos na captação de doadores nas tecnologias existentes e ao contexualizar no cenário Pernambucano, as dificuldades encontradas em um software robusto neste processo de engajamento de pessoas aptas a doar sangue, não é diferente. Com isso, a proposta do 1 upDonate tem como motivação, desenvolver um software gamificado para resolver o problema na baixa de coleta de sangue. A aplicação é integrada à mídia social Facebook e está disponível nas plataformas Android, iOS e Web.

Os requisitos dos módulos web e mobile foram desenvolvidos para garantir uma versão executável em um ambiente de produção, ou seja, no dia-a-dia dos profissionais dos hemocentros; pode-se, com isso, disponibilizar mais um canal de acesso ao doador, fazendo com que os bancos de sangue se beneficiem com as aplicações 1upDonate mobile e web.

Como trabalhos futuros propõe-se a validação do protótipo, para que o público alvo dê feedback com relação as questões de design e usabilidade, fatores relevantes para uma boa aceitação de um software.

Um requisito bastante interessante para implementar, em novos trabalhos, é a recomendação inteligente baseada na proximidade da residência dos usuários com os hemocentros e o recebimento de um push notification de uma campanha cadastrada quando o solicitante do sangue for do mesmo tipo sanguíneo e esteja em uma região próxima ao doador, ou seja, o sistema automaticamente elencaria os potenciais doadores pela geolocalização.

Outras melhorias mais simples também podem ser implementadas, como a otimização do processo de login para o Google+ e Twitter; a possibilidade do doador cadastrar os hemocentros no app e no portal, ter algum gerenciador para apenas corrigir estes dados e a inclusão de filtros para facilitar as buscas dos dados.

Num contexto mais abrangente, o 1upDonate poderá ser aplicado a contextos como a doação de medula óssea ou até mesmo em uma atmosfera completamente fora da área de saúde, pois os conceitos de gamificação são genéricos e sem restrição de uso.

A aplicação móvel foi criada de maneira híbrida e com isso, é possível também gerar a mesma versão para o sistema operacional iOS, no entanto, isso não foi compilado e testado. Seria interessante tratar essa outra tecnologia.

Após os testes, as versões para Android e iOS poderiam ser disponibilizadas nas lojas de aplicativos para que sejam elencados alguns usuários pilotos para validar o 1upDonate na prática e com isso, verificar as possíveis melhorias que não foram enxergadas no contexto deste artigo.

\section{Referências}

[1] Gonçalez, Thelma; C. Sabino, Ester; F. Chamone1, Dalton. Trends in the profile of blood donors at a large blood center in the city of São Paulo, Brazil, p. 144-148, 2013.

[2] Ingrid Souza Barboza, Stephanie; José da Costa, Francisco.Marketing social para doação de sangue: análise da predisposição de novos doadores, p. 1463-1474, jul, 2014.

[3] OMS: doações de sangue precisam aumentar em mais da metade dos países. http://agenciabrasil.ebc.com.br/internacional/not icia/2016-06/oms-diz-que-doacoes-voluntariasde-sangueprecisam-aumentar. Acessado em: 27/09/2016.

[4] OMS afirma que doações de sangue precisam aumentar em mais da metade dos países. http://www.uai.com.br/app/noticia/saude/2016/ 06/14/noticias-saude, $190050 /$ oms-afirma-quedoacoes-de-sangue-precisam-aumentar-em- 
mais-da-metade-d.shtml. Acessado em: 27/09/2016.

[5] Moraes, Éder Junio;Freitas Moreira, Rossana.Ferramenta para gestão de hemocentros com aplicativo para divulgação de doações de sangue no Facebook, Jul, 2015.

[6] Colling, Ilseu Luís; André da Costa, Cristiano; da Righi, Rodrigo. Um Modelo Ubíquo para Doação de Sangue baseado em Dispositivos Móveis, 2014.

[7] A Bhowmik, N A Nabila, M A Imran, M A U Rahman and D Karmaker. An Extended Researchon the Blood Donor Community as a Mobile Application. p. 26-34, 2015.

[8] Stadler, Dorothe; Bilgram, Volker. Gamification: Best Practices in Research and Tourism. p. 363-370,2016.

[9] Fotopoulos, Ilias; Palaiologou, Revekk, Kouris, Ioannis; Koutsouris, Dimitrios. Cloud-Based Information System for Blood Donation. p. 797$801,2016$.

[10] S. Abu Naser, Samy; Zaqout, Ihab. Design and Development of Mobile Blood Donor Tracker. p. 294- 300, fev, 2016.

[11] Software blood donor https://play.google.com/store/apps/details?id=co m.cube.arc.blood. Acessado em: 29/09/2016.

[12] Apple X-Code: https://developer.apple.com/xcode/. Acessado em: 28/11/2016.

[13] Robustez Jboss: http://blog.cvinicius.com.br/2013/06/jboss-as-7instalacao-e-configuracao.html. Acessado em: 28/11/2016.

[14] Lei do Sangue; Lei Betinho: http://www2.camara.leg.br/legin/fed/lei/2001/lei -10205-21-marco-2001-364841-norma-pl.html. Acessado em: 29/11/2016.

[15] Ouhbi, Sofia; Fernández-Alemán, Jose Luis; Toval, Ambrosio; Idri, Ali; Pozo, Jose Rivera. Free Blood Donation Mobile Applications. p. 1-20, 2015.

[16] Push_notification:

http://searchmobilecomputing.techtarget.com/de 59 finition/ push-notification. Acessado em: 29/11/2016.

[17] Vianna, Ysmar; Vianna, Maurício; Medina,Bruno; Tanaka, Samara. Gamification, Inc. Como reinventar empresas a partir de jogos. 2015.

[18] Nike+FuelBand:

https://play.google.com/store/apps/details?id=co m.nike.fb\&hl=pt_BR. Acessado em: 29/11/2016.

[19] Foursquare:

https://play.google.com/store/apps/details?id=co $\mathrm{m}$.joelapenna.foursquared\&hl=pt_BR. Acessado em: 29/11/2016.

[20] Runkeeper:

https://play.google.com/store/apps/details?id=co $\mathrm{m}$. fitnesskeeper. runkeeper. pro\&hl=ptBR.

Acessado em: 29/11/2016.

[21] McGonigal,Jane: A Realidade Em Jogo - Por Que Os Games Nos Tornam Melhores e Como Eles Podem Mudar o Mundo. 2012.

[22] Junior, Gilson Cruz:

www.revista.ueg.br/index.php/praxia/article/dow nload/1431/946. acessado em: 29/11/2016.

[23] Pohl, Klaus; Rupp, Chris. Fundamentos da Engenharia de Requisitos. p. 30. 2013.

[24] Pressman, Roger S: Engenharia de Software, $7^{a}$ edição. p. 727. 2011.

[25] Elmasri, Navathe: Engenharia de Software, 7 edição. p. 38. 2010. 